\title{
Sex-related differences in continuous positive airway pressure adherence
}

\author{
Jun Shitara ${ }^{1} \cdot$ Takatoshi Kasai $^{2,3,4}$ \\ Published online: 26 February 2022 \\ (c) The Author(s), under exclusive licence to Japanese Society of Sleep Research 2022
}

Obstructive sleep apnea (OSA) is a highly prevalent chronic disorder worldwide [1], observed in $10-20 \%$ of the general population [2]. OSA has been recognized as a major disease in men for several years [3]; however, recent reports have indicated that OSA may also have a high prevalence in women, and that there are sex-related differences in the age of onset, rate of diagnosis, sleep characteristics, and therapeutic effects [4]. Current population-based studies, including data from Japan, suggest that in addition to men, a substantial proportion of women, especially postmenopausal women, suffer from OSA [2]. It is estimated that up to $90 \%$ of women with severe sleep apnea are not diagnosed, and if diagnosed, are less likely to be treated [5]. One of the causes of this deficit is that some of the common questionnaires used to determine sleepiness, such as the Epworth Sleepiness Scale, are more sensitive in men than in women [4]. The other cause is less frequent sleep testing, such as testing with polysomnography, which is performed in women with complaints of insomnia or poor sleep rather than sleepiness [6]. Sex-related differences have been reported in apnea during rapid eye movement sleep, and electroencephalogram arousals from sleep are more common in women than in men [7].

Continuous positive airway pressure (CPAP) is considered the gold standard therapy for moderate-to-severe OSA

Takatoshi Kasai

kasai-t@mx6.nisiq.net

1 Department of Cardiovascular Medicine, Juntendo Shizuoka Hospital, 1129 Nagaoka, Izunokuni City, Shizuoka 410-2295, Japan

2 Cardiovascular Respiratory Sleep Medicine, Juntendo University Graduate School of Medicine, Tokyo, Japan

3 Sleep and Sleep Disordered Breathing Center, Juntendo University Hospital, Tokyo, Japan

4 Department of Cardiovascular Biology and Medicine, Juntendo University Graduate School of Medicine, 3-1-3 Hongo, Bunkyo-ku, Tokyo, Japan and remains the most effective treatment. Thus, it is important to understand the factors that influence CPAP adherence to maintain high adherence. Previous reports have shown that factors including economic characteristics, disease severity, psychological factors, and side effects are thought to affect CPAP adherence [8]. In addition, Campos-Rodriguez et al. reported that a greater frequency of apneas and hypopneas (i.e., apnea-hypopnea index: AHI) and a greater degree of hypoxia are associated with better CPAP adherence even in non-sleepy patients, suggesting that patients with severe OSA are likely to show good adherence, regardless of sleepiness [9]. Few clinical studies have assessed sexrelated differences in the factors influencing CPAP adherence. However, Fujita et al. [10] did investigate sex-related differences in the factors influencing CPAP adherence. In their study, neither AHI nor indices of hypoxia were associated with CPAP adherence in women, whereas a greater AHI was associated with better CPAP adherence in men. Although similar findings have been reported in a European patient population [11], the findings of the study by Fujita et al. provide important clinical information for the care of CPAP users in Japan.

Regarding the factors following the initiation of CPAP, Fujita et al. reported that some complaints, such as insomnia and/or lack of deep sleep, were significantly associated with poor adherence, while improved daytime sleepiness was significantly associated with good adherence in both men and women. In men, other complaints may also influence CPAP adherence; respiratory difficulty and maladaptation to CPAP were negatively associated with adherence, while decreased awakenings, reduced nocturia, and adaptation to CPAP positively influenced adherence. However, no significant associations between other complaints and CPAP adherence were found in women. Thus, in Japan, sleep physicians who are monitoring patients with CPAP should consider these findings, especially when enquiring about complaints and difficulties following CPAP initiation. 
A limitation leaves several questions regarding the interpretation of these findings. As these findings were obtained from surveillance of a single academic sleep center located in one of the biggest cities in Japan, the enrolled patients may not represent the typical Japanese patient population with OSA. Thus, a multicenter study or national survey is required to draw solid conclusions.

Funding None.

\section{Declarations}

Conflict of interest T. Kasai is affiliated with departments endowed by Philips Respironics, ResMed, Teijin Home Healthcare and Fukuda Denshi, Paramount Bed, Philips, Asahi Kasei, InterReha, and Toho Holdings.

\section{References}

1. Senaratna CV, Perret JL, Lodge CJ, Lowe AJ, Campbell BE, Matheson MC, et al. Prevalence of obstructive sleep apnea in the general population: a systematic review. Sleep Med Rev. 2017;34:70-81.

2. Matsumoto T, Chin K. Prevalence of sleep disturbances: sleep disordered breathing, short sleep duration, and non-restorative sleep. Respir Investig. 2019;57:227-37.

3. Block AJ, Boysen PG, Wynne JW, Hunt LA. Sleep apnea, hypopnea and oxygen desaturation in normal subjects. A strong male predominance. N Engl J Med. 1979;300:513-7.
4. Kumar S, Anton A, D'Ambrosio CM. Sex differences in obstructive sleep apnea. Clin Chest Med. 2021;42:417-25.

5. Lindberg E, Benediktsdottir B, Franklin KA, Holm M, Johannessen A, Jögi R, et al. Women with symptoms of sleep-disordered breathing are less likely to be diagnosed and treated for sleep apnea than men. Sleep Med. 2017;35:17-22.

6. Auer M, Frauscher B, Hochleitner M, Högl B. Gender-specific differences in access to polysomnography and prevalence of sleep disorders. J Womens Health (Larchmt). 2018;27:525-30.

7. O'Connor C, Thornley KS, Hanly PJ. Gender differences in the polysomnographic features of obstructive sleep apnea. Am J Respir Crit Care Med. 2000;161:1465-72.

8. Mehrtash M, Bakker JP, Ayas N. Predictors of continuous positive airway pressure adherence in patients with obstructive sleep apnea. Lung. 2019;197:115-21.

9. Campos-Rodriguez F, Martinez-Alonso M, Sanchez-de-la-Torre M, Barbe F. Long-term adherence to continuous positive airway pressure therapy in non-sleepy sleep apnea patients. Sleep Med. 2016;17:1-6.

10. Fujita K, Chishaki H, Ando Si, et al. Sex differences in the effectiveness and affecting factors to adherence of continuous positive airway pressure therapy. Sleep Biol Rhythms. 2022. https://doi. org/10.1007/s41105-021-00355-4.

11. Campos-Rodriguez F, Martinez-Garcia MA, Reyes-Nuñez N, Caballero-Martinez I, Almeida-Gonzalez CV, Catalan-Serra P, Peña-Griñan N. Long-term continuous positive airway pressure compliance in females with obstructive sleep apnoea. Eur Respir J. 2013;42:1255-62.

Publisher's Note Springer Nature remains neutral with regard to jurisdictional claims in published maps and institutional affiliations. 